$\stackrel{W}{=}$

Global burnals Inc.

8

\title{
Inter-Observer Variation in the Grading of Meningiomas using the WHO Classification of CNS Tumors Criteria
}

By Hisham Alkhalidi

King Saud University

Abstract- Background: Grading of meningiomas using the World health organization (WHO) Classification of the Central Nervous System criteria currently has an essential role in classification, treatment, prognosis prediction, and research of these tumors.

Aims: This is a retrospective study that assessed the interobserver variation between Anatomical Pathologists in grading meningiomas using material obtained from ten resection specimens. The WHO grading system includes different methods, including the mitotic count, the tumor subtypes or the presence of three out of five certain morphological features. This paper focuses on the interobserver variability in the latter method.

Methods: Meningiomas that were originally graded based upon mitoses, brain invasion, or morphological subtype were excluded. Ten different Anatomical Pathologists, including two Neuropathologists, who were blinded to the original diagnosis and grade graded the tumors independently.

Keywords: meningioma, radiation therapy, interobsenver variation.

\section{GJMR-C Classification: NLMC Code: QW 4}

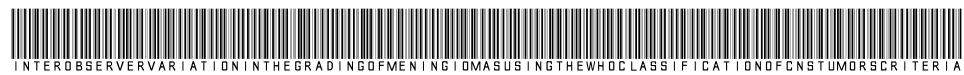

Strictly as per the compliance and regulations of:

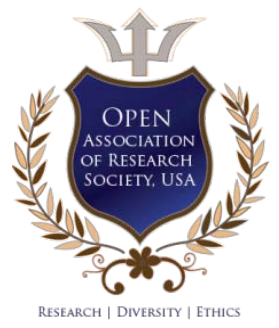

(C) 2020. Hisham Alkhalidi. This is a research/review paper, distributed under the terms of the Creative Commons AttributionNoncommercial 3.0 Unported License http://creativecommons.org/licenses/by-nc/3.0/), permitting all non-commercial use, distribution, and reproduction in any medium, provided the original work is properly cited. 


\title{
Inter-Observer Variation in the Grading of Meningiomas using the WHO Classification of CNS Tumors Criteria
}

\author{
Hisham Alkhalidi
}

\begin{abstract}
Background: Grading of meningiomas using the World health organization (WHO) Classification of the Central Nervous System criteria currently has an essential role in classification, treatment, prognosis prediction, and research of these tumors.

Aims: This is a retrospective study that assessed the interobserver variation between Anatomical Pathologists in grading meningiomas using material obtained from ten resection specimens. The $\mathrm{WHO}$ grading system includes different methods, including the mitotic count, the tumor subtypes or the presence of three out of five certain morphological features. This paper focuses on the interobserver variability in the latter method.
\end{abstract}

Methods: Meningiomas that were originally graded based upon mitoses, brain invasion, or morphological subtype were excluded. Ten different Anatomical Pathologists, including two Neuropathologists, who were blinded to the original diagnosis and grade graded the tumors independently.

Results: There was "intermediate to good" interobserver agreement between the Pathologists using this method. The kappa score for interobserver agreement between the ten Anatomical Pathologists was 0.53 , and between the two Neuropathologists was 0.55 , with an overall agreement percentage of $70 \%$.

Conclusions: More precise grading criteria and definitions can improve the interobserver agreement. Clinicians and researchers need to understand the difficulty in grading some meningiomas.

Keywords: meningioma, radiation therapy, interobserver variation.

\section{INTRODUCTION}

$\mathrm{M}$ eningiomas are relatively common dura-based tumors that constitute about $25-30 \%$ of primary brain tumors in Saudi Arabia $(1,2)$. Grading of meningiomas has an essential role in patient management, including classification, treatment and prognosis prediction. Tumors with a higher grade have more chances of increased recurrence and mortality rates (3). The treatment of atypical and anaplastic meningioma is based on surgery and radiation therapy (4). The updated 2016 edition of the World Health Organization (WHO) Classification of Tumours of the Central Nervous system (5) provides the criteria used in

Corresponding Author: Associate Professor, Department of Pathology, College of Medicine, King Saud University.

e-mail: hishamkh@gmail.com the classification of meningiomas into three grades (from grade I to grade III). These criteria include the subtype of the tumor, brain invasion or mitotic counts, in addition to the presence of an overtly malignant highgrade morphology (i.e. sarcomatoid, carcinomatoid or melanoma). If three out of specific five criteria are present in a given meningioma, it should be graded as grade II. These five criteria are increased cellularity, small cells change with high N/C ration, large and prominent nucleoli, patternless or sheet-like growth and foci of spontaneous or geographic necrosis. These criteria do not clarify a quantitative definition. This paper explores the responses of ten pathologists who examined ten cases of meningiomas, where grading variability may occur due to different interpretations of these five criteria.

\section{METHOD}

In this retrospective study, forty-two meningioma cases were retrieved from the archives of the histopathology unit at King Khalid University Hospital, Riyadh, from 2017 to 2019. Excluded cases included any atypical or anaplastic meningiomas that showed brain invasion or were graded based on the increased mitotic count. Any meningioma that was graded as grade II or grade III based on the morphological subtype was also excluded. These subtypes are clear cell, chordoid, papillary and rhabdoid. Five grade I meningiomas were randomly selected, based on the original pathology report. Another five grade II meningiomas were randomly selected. The number of blocks for each case varied from one to fourteen, which can be attributed to the variation in the volume of tumor tissue submitted for histopathological assessment. All biopsies were processed and stained using routine hematoxylin and eosin stain. An experienced Neuropathologist reviewed each case and selected a representative slide. Ten experienced Anatomical Pathologists reviewed the selected slides and recorded the grade. The pathologists include two experienced Neuropathologists. The review was performed without knowledge of the previous clinical, radiological, or histopathological findings of the ten patients from whom those biopsies were obtained. A detailed educational 
sheet was used, expecting each pathologist to follow the WHO (2016) criteria for Meningioma grading. The results obtained from each pathologist were independently documented as meningioma (grade I), atypical meningioma (grade II) or anaplastic meningioma (grade III). A Neuropathologist recorded the morphological features of the ten cases. The results were tabulated and analyzed by the multiple-reader Cohen kappa statistical analysis method using a website based calculator (6) and a free-marginal multirater kappa. The aim was to assess the precision of agreement between the various observers (interobserver agreement).

\section{RESULTS}

The results obtained by the ten participating pathologists and the two neuropathologists are summarized in Table 1. The kappa score for interobserver agreement between the ten anatomical pathologists was $0.53(95 \% \mathrm{Cl}$ for free-marginal kappa $[0.32,0.74]$ ) with an overall agreement percent of $68.44 \%$. The kappa score for interobserver agreement between the two neuropathologists was $0.55(95 \% \mathrm{Cl}$ for free-marginal kappa 0.1 , and 1) with an overall agreement percent of $70 \%$. Both kappa scores are in keeping with above chance "intermediate to good" agreement (7). The cases that showed $100 \%$ agreement were three cases. Two cases were $\mathrm{WHO}$ grade I. The third case was WHO grade II (Case 2, Figure 1). The latter was the only meningioma from the selected cases that showed focal necrosis. Another five cases were graded as either WHO grade I or WHO grade II by the reviewers. These cases were showing a variable degree of small cell change, lack of pattern, and cellularity (Figure 2). These cases did not show features of necrosis or prominent nucleoli. Two cases (Cases 8 and 9) were labelled as WHO grade II by the majority of the reviewers. However, in each one of them, two reviewers labelled them as WHO grade III (Figure 3). In their opinion, the reason for such designation was the focal presence of sarcomatoid morphology.

\section{Discussion}

Meningiomas grading has an essential role in patients management and related research studies (8). The risk of recurrence further increases with WHO grade. In one study, patients with benign, atypical, and malignant meningiomas had a 10-year cumulative incidence of recurrence of $6 \%, 17 \%$, and $30 \%$, respectively. The 10-year relative survival of patients with WHO grade I, II, and III meningiomas were 97\%, 90\%, and $30 \%$, respectively. These numbers demonstrate the significant increase in tumor-related mortality based upon the WHO grade (3). There is no clear recommendation about the use of radiation therapy in meningiomas (9). However, the higher grade the tumor, the more chances that the patient will receive adjuvant therapy (e.g., External Beam Radiotherapy). Surgical resection extent is the most important prognostic factor among malignant meningioma patients $(3,10)$. The extended safety margins are necessary to achieve a favorable local control for high-grade meningiomas (11). 15 to $80 \%$ improvement of the 5-year progression-free survival was reported when RT was added to surgical resection for malignant meningioma. Atypical meningiomas appear to be more frequently diagnosed under the WHO classification system updates (12). No consensus exists for "atypical" meningiomas treatment, and radiation therapy has mostly been reserved for recurrence and progression (13,14). Gross total resection and adjuvant radiation therapy appear to be highly associated with improved survival, independent of other factors, in patients with atypical meningiomas (15). Overall, the grading of meningiomas is essential and has a significant impact on both the clinical research studies and the treatment of these tumors.

Among the ten practicing pathologists and the two neuropathologists, this study's findings show that the inter-observer agreement on the grading of meningioma that is based upon the presence of the specified three out five features is "intermediate to good" above chance. Clinicians and researchers should be aware of this issue and the subjectivity element in the grading criteria. Three cases out of ten had a perfect agreement. Seven cases had discrepancies, while five cases were graded either as grade I or grade II, and two cases were essentially graded as grade II or grade III.

This paper does not incorporate grade II and grade III meningiomas that were classified based on relatively more objective criteria, including the brain invasion or the specific tumor subtype Morphology. Similar studies are limited in this field. In one study, the mitotic count is considered an objective method of grading, but variation in the grading using mitoses has been reported between pathologists based upon the number of fields examined (16). Another study (17) using previous $\mathrm{WHO}$ versions showed high concordance between the pathologists for brain invasion, $\geq 20$ mitoses/10 high -powered field and spontaneous necrosis. The concordance was lowest for small cells, sheeting and $\geq 4$ mitoses/10 HPF. For atypical meningioma, the criteria of diagnosis include the presence of the three out of five morphological features (3 out 5), as mentioned earlier. The case that displayed necrosis was the only one that had a perfect agreement as grade II. It appears that necrosis presence was a feature that prompted all the pathologists to look for more needed features to label the tumor as grade II during the case screening. However, the rest of the five features appears to be more problematic. The WHO criteria do not state the percentage of the tumor area, showing the features that are needed to apply criteria. Furthermore, the features 
are needed to apply criteria. Furthermore, the features do not have a quantitative definition. For example, regarding "increased cellularity", the criteria do not mention how many cells are needed per space unit to consider the tumor cellular. For "small cell change", the $\mathrm{N}: \mathrm{C}$ ratio that should be present to consider a tumor cell as a small cell is not mentioned. "Prominent nucleoli" is left for the pathologist's judgments. In other body systems, the magnification power is used as a quantitative method for defining visible, prominent nucleoli (18).

For grade III tumors, one of the defining grading methods is the presence of sarcomatoid or carcinomatoid morphology. No statement of the volume of the tumor that should show this feature is clarified in the WHO criteria. Besides, these patterns may have a room for personal interpretation and opinions diversity. This explains the two cases where two pathologists labeled them as grade III, while the majority of the remaining pathologists graded them as grade II.

\section{Conclusion}

Atypical and anaplastic meningiomas can be challenging diseases, not only from a treatment perspective but also from a diagnosis perspective. As demonstrated, the current Meningioma grading system provided by the WHO book does not draw a sharp line between the different grade categories in a significant subset of meningiomas, and more precise criteria and definitions can help. This issue is particularly applicable to the following features: cellularity, lack of pattern, small cell change, prominent nucleoli and sarcomatoid or carcinomatoid morphology. A significant difference in interpretations may make it difficult to establish a definitive cut off that would translate accurately from one laboratory to another. Hence, clinicians and researchers should be aware of this concern. Understanding the grading criteria and the pathology report and communication with the pathologist are an essential element of meningiomas management. These tumors are signed out by general Anatomical Pathologists in many places. Hence, they should also be aware of the meningioma grading criteria and related-concerns. The pathology reports should include the basis of the grading and any difficulty that is associated with it. Besides, intradepartmental consultations, Pathologist's education and joint reporting by two experienced pathologists can help to maintain a high level of grading concordance. The biological signature of meningiomas is likely to play a significant role in the evolution of the grading system strategy. Hopefully, studies focusing on the immunohistochemical and genetic features of these complex tumors and relating these features to the treatment response and the prognosis will provide a more reproducible system with better concordance between pathologists and laboratories.

\section{ACKNOWLEDGMENTS}

The author would like to express his gratitude to all the pathologists who kindly participated in this study.

\section{References Références Referencias}

1. Almutrafi A, Bashawry $Y$, AlShakweer W, Al-Harbi M, Altwairgi A, Al-Dandan S. The Epidemiology of Primary Central Nervous System Tumors at the National Neurologic Institute in Saudi Arabia: A TenYear Single-Institution Study. J Cancer Epidemiol. 2020; 2020:1429615. 15 Feb. 2020, doi:10.1155/ 2020/1429615

2. Mohammed AA, Hamdan AN, Homoud AS. Histopathological Profile of Brain Tumors: A 12-year Retrospective Study from Madinah, Saudi Arabia. Asian J Neurosurg. 2019; 14(4):1106-1111. doi:10.4103/ajns.AJNS 18519

3. Holleczek B, Zampella D, Urbschat S,Sahm F, von Deimling A, Oertel J, et al. Incidence, mortality, and outcome of meningiomas: A population-based study from Germany. Cancer Epidemiol. 2019; 62:101562. doi:10.1016/j.canep.2019.07.001

4. Apra C, Peyre M, Kalamarides M. Current treatment options for meningioma. Expert Rev Neurother. 2018; 18(3):241-249. doi:10.1080/14737175.2018. 1429920

5. Louis DN, Ongaki H, Wiestler OD, Cavenee WK. WHO Classification of Tumours of the Central Nervous System. Lyon: World Health Organization: 2016.

6. Randolph J. (2008). Online Kappa Calculator [Computer software]. (http://justus.randolph.name/ kappa, accessed 1 June 2020).

7. Fleiss, J. L. Statistical methods for rates and proportions. Hoboken, JN: Wiley: 1981.

8. Dobran M, Marini A, Splavski B, Rotim K, Liverotti V, Nasi $D$, et al. Surgical treatment and predictive factors for atypical meningiomas: a multicentric experience. World Neurosurg. 2020; S18788750(20)30672-0. published online ahead of print, 2020 Apr 17, doi:10.1016/j.wneu.2020.03.201

9. Poulen $G$, Vignes JR, Le Corre $M$, Loiseau $H$, Bauchet L. WHO grade II meningioma: Epidemiology, survival and contribution of postoperative radiotherapy in a multicenter cohort of 88 patients. Neurochirurgie. 2020; 66(2):73-79. doi:10.1016/j.neuchi.2019.12.008

10. Nanda A, Bir SC, Konar S, Maiti T, Kalakoti $P$, Jacobsohn JA, etal. Outcome of resection of WHO grade II meningioma and correlation of pathological and radiological predictive factors of recurrence. Journal of Clinical Neuroscience. 2016; 31:112-121. DOI: 10.1016/j.jocn.2016.02.021

11. Zollner B, Ganswindt U, Maihöfer C, Corradini S, Albert N, Schichor C. et al. Recurrence pattern analysis after [68Ga]-DOTATATE-PET/CT -planned 
radiotherapy of high-grade meningiomas. Radiat Oncol. 2018; 13(1):110. doi:10.1186/s13014-0181056-4

12. Willis J, Smith C, Ironside JW, Erridge S, Whittle IR, Everington $\mathrm{D}$. The accuracy of meningioma grading: a 10-year retrospective audit. Neuropathol ApplNeurobiol. 2005; 31(2):141-149. doi:10.1111/j. 1365-2990.2004.00621.x

13. Dziuk TW, Woo S, Butler EB, Thornby J, Grossman R, Dennis WS, et al. Malignant meningioma: an indication for initial aggressive surgery and adjuvant radiotherapy. J Neurooncol. 1998; 37(2):177-188. doi:10.1023/a:1005853720926

14. Jung GS, Ramina R, da Silva Jr EB, Coelho Neto M. Diagnosis and Predictors of Treatment in Atypical and Anaplastic Meningiomas. Jornal Brasileiro de Neurocirurgia. 2018; 27 (2): 100 -14. doi: https://doi. org/10.22290/jbnc.v27i2.751

15. Rydzewski NR, Lesniak MS, Chandler JP, Kalapurakal JA, Pollom E, Tate MC, et al. Gross total resection and adjuvant radiotherapy most significant predictors of improved survival in patients with atypical meningioma. Cancer. 2018; 124(4): 734-742. doi:10.1002/cncr.31088

16. Saygin I, Cakir E, Ercin ME. Interobserver Variability in Mitotic Count for Meningioma Grading: How Can We Reduce It?. Turk Neurosurg. 2019; [published online ahead of print, 2019 Jul 11], doi:10.5137/ 1019-5149.JTN.26252-19.2

17. Rogers CL, Perry A, Pugh S, Vogelbaum MA, Brachman D, McMillanW, et al. Pathology concordance levels for meningioma classification and grading in NRG Oncology RTOG Trial 0539. Neuro Oncol. 2016; 18(4):565-574. doi:10.1093/ neuonc/nov247

18. Delahunt B, Cheville JC, Martignoni G, Humphrey PA, Magi-Galluzzi C, McKenney $J$, et al. The International Society of Urological Pathology (ISUP) grading system for renal cell carcinoma and other prognostic parameters. Am J SurgPathol. 2013; 37(10):1490-1504. doi:10.1097/PAS.0b013e318 $299 f 0 f b$

Table 1: Grading responses of ten different meningiomas samples by ten Anatomical Pathologists (AP) including two Neuropathologists (NP)

\begin{tabular}{|c|c|c|c|c|c|c|c|c|c|c|}
\hline Cases & NP & NP & AP & AP & AP & AP & AP & AP & AP & AP \\
\hline 1 & 1 & 1 & 1 & 1 & 1 & 1 & 1 & 1 & 1 & 1 \\
\hline 2 & 2 & 2 & 2 & 2 & 2 & 2 & 2 & 2 & 2 & 2 \\
\hline 3 & 1 & 2 & 1 & 1 & 2 & 2 & 1 & 2 & 1 & 1 \\
\hline 4 & 1 & 1 & 1 & 1 & 1 & 1 & 1 & 1 & 1 & 1 \\
\hline 5 & 2 & 2 & 2 & 2 & 1 & 1 & 2 & 2 & 1 & 2 \\
\hline 6 & 1 & 1 & 1 & 1 & 1 & 1 & 2 & 2 & 1 & 1 \\
\hline 7 & 1 & 1 & 1 & 1 & 1 & 1 & 2 & 2 & 1 & 2 \\
\hline 8 & 2 & 2 & 2 & 2 & 2 & 2 & 2 & 3 & 2 & 3 \\
\hline $\boldsymbol{9}$ & 3 & 2 & 2 & 2 & 1 & 2 & 2 & 3 & 2 & 2 \\
\hline 10 & 1 & 2 & 1 & 1 & 1 & 1 & 2 & 2 & 1 & 1 \\
\hline
\end{tabular}

Figures Legends:

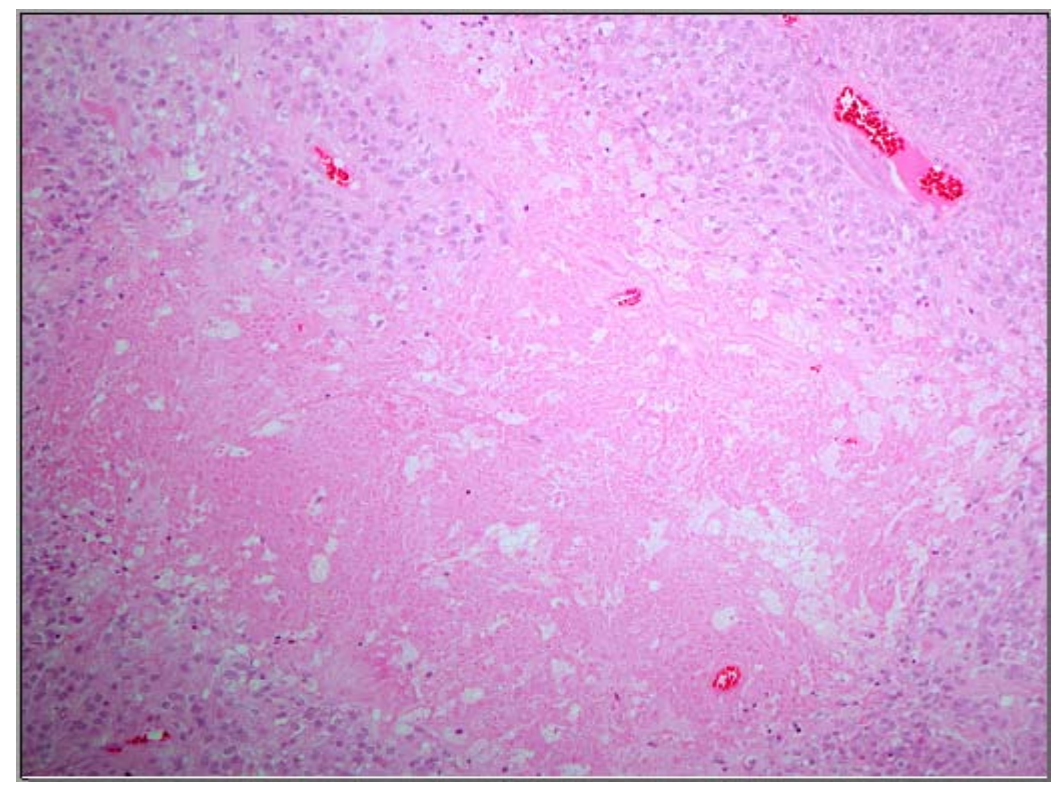

Figure 1: Necrosis in a grade II meningioma. The surrounding viable meningioma tissue shows cellularity and lack of pattern. (H\&E X200). 


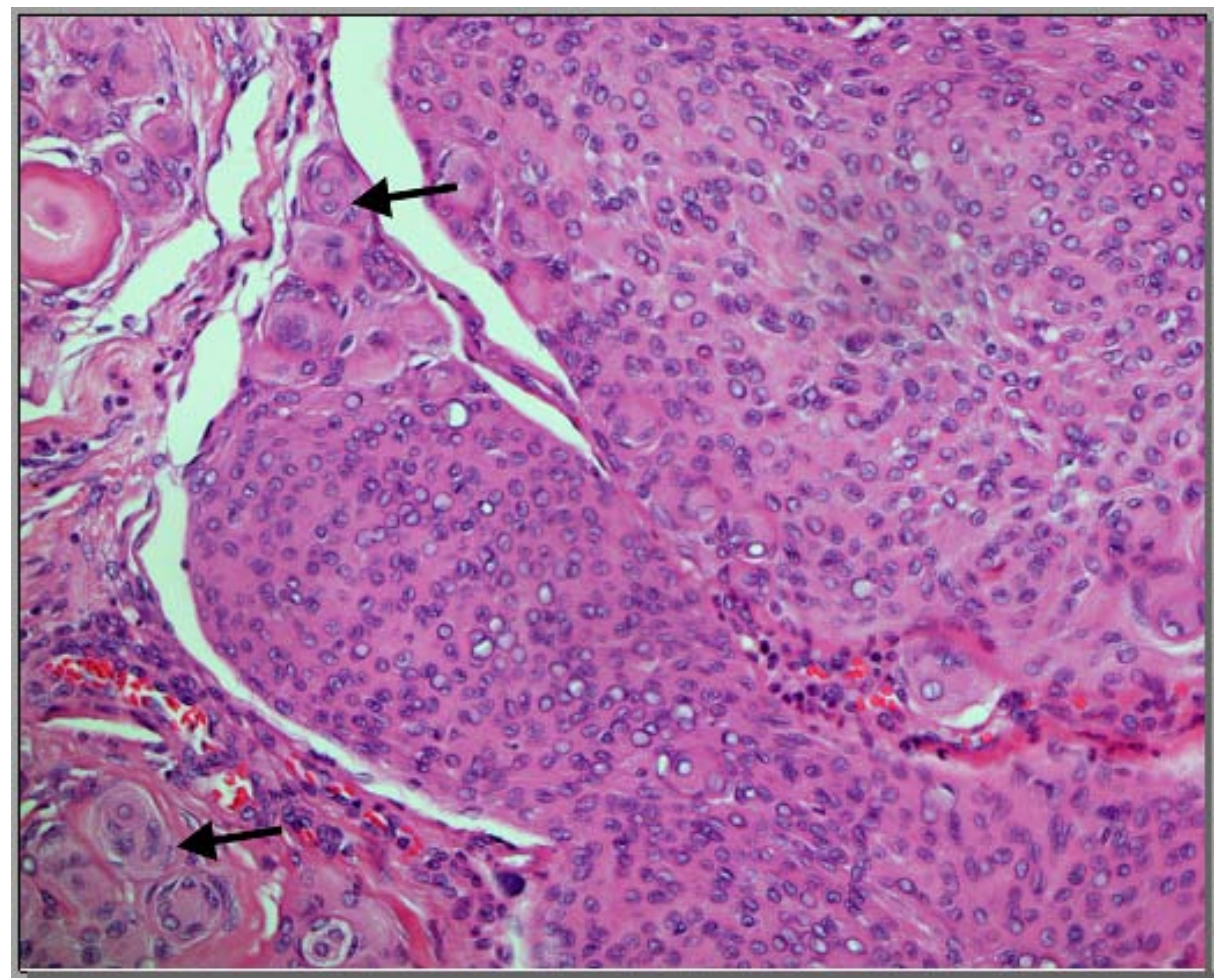

Figure 2: A meningioma showing cellular areas, foci of increased N: C ratio and partial lack of pattern. In the same field, meningothelial pattern with prominent whorls (arrows) is present (H\&E X200).

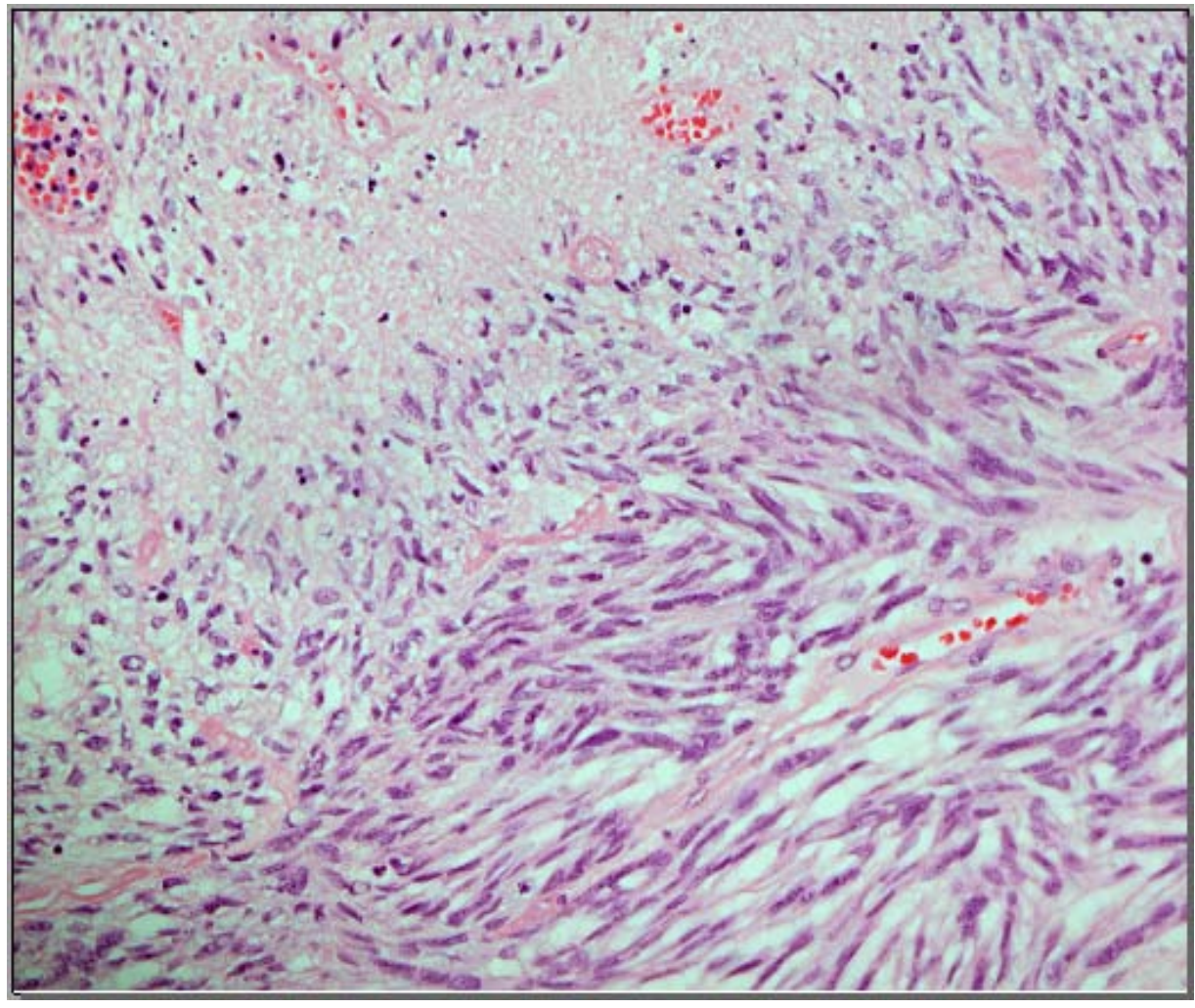

Figure 3: A meningioma showing focal necrosis and adjacent cellular proliferation of spindle cells with increased $\mathrm{N}$ :

$\mathrm{C}$ ratio and foci of small cell change. Two pathologists considered such foci to be "sarcomatoid" (H\&E, x200). 